Peter Anderson, MD, FRCSC

\section{Président, AUC}

Cite as: Can Urol Assoc J 2014;8(5-6):157.

http://dx.doi.org/10.5489/cuaj.2183

Published online June 16, 2014.

\section{L'AUC a pour mandat de} promouvoir les plus hautes normes dans les soins urologiques pour les Canadiens et de faire avancer l'art et la science de l'urologie.

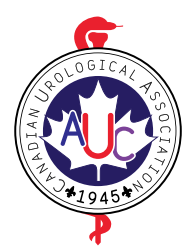

I y a près d'un an, je signalais dans ces pages notre intention de revoir l'orientation stratégique de I'AUC'1. Depuis, les membres du comité exécutif et d'autres intervenants clés ont travaillé sans relâche pour mettre sur pied ce que nous considérons être un plan d'action pratique et visionnaire qui guidera I'AUC au cours des cinq prochaines années. Ce plan a été délibérément conçu selon un processus itératif, sachant que ses jalons sont en constante évolution et que nous devrons faire preuve de souplesse en réaction à la transformation de notre environnement. Au moment où j'écris ces lignes, nous en sommes à la dernière étape du processus consultatif. Pendant cette phase, les membres de l'AUC seront sondés en fonction de trois axes précis :

1. Quels ont été nos bons coups ?

2. Quels ont été nos ratés?

3. Que pouvez-vous faire pour contribuer à la mise en œuvre du plan d'action ?

Notre stratégie se résume en trois énoncés de mission et un objectif complémentaire essentiel :

1. Accroître la mobilisation des membres.

L'AUC prend appui sur l'action bénévole, et nos activités doivent reposer sur des moyens concrets pour répondre efficacement aux besoins de nos membres. La Fondation des bourses de l'AUC (FBAUC) constitue à cet égard un acquis précieux. Nous examinerons les façons d'élargir la portée de l'offre faite aux membres, même au-delà du cadre strict de la recherche universitaire. Nous nous emploierons à élaborer des outils de pratique utiles pour nos membres et nous nous pencherons sur diverses initiatives telles que la création d'un programme de mentorat mettant en relation les nouveaux diplômés avec des urologues bien établis.

2. Être la voix de l'urologie auprès des responsables des politiques canadiennes en matière de santé.

À l'heure où l'on se dispute de plus en plus les ressources en soins de santé, I'AUC est appelée à jouer un rôle de premier plan dans la défense des intérêts des patients et de notre profession. Afin de mieux servir cette mission, nous prévoyons revoir le mandat du comité des affaires socioéconomiques, qui prendra le nom de comité des politiques en santé afin de refléter plus fidèlement ses responsabilités. Nous devrons également plaider en faveur de l'établissement d'un nombre suffisant de postes de formation à l'échelle du pays.

3. Être la ressource canadienne par excellence en urologie pour les patients et les professionnels de la santé.

L'AUC jouit déjà d'une importante visibilité à ce chapitre, mais il y a place à I'amélioration et à la collaboration, notamment avec les autres professionnels de la santé et les groupes de défense des intérêts des patients.

4. Assurer la viabilité financière de I'AUC.

L'ensemble des objectifs qui précèdent dépendent de la santé financière continue de l'AUC. Nous devons examiner les solutions innovatrices qui s'offrent à nous afin de préserver et de renforcer les ressources budgétaires requises pour mener à bien notre plan stratégique.

L'orientation stratégique de l'AUC énoncée dans le plan se veut une feuille de route dynamique. Il importe ici de rappeler les mots de l'écrivain français et pionnier de l'aviation Antoine de Saint-Exupéry : "Tout objectif sans plan n'est qu'un souhait ». Je fais appel à vous pour épauler l'organisation de la manière qui vous paraîtra la plus efficace, car seule la mobilisation de nos membres peut nous permettre d'atteindre nos buts.

\section{Reference}

1. Anderson P. What has the CUA done for you lately? Can Urol Assoc J 2013;7:303. 